\section{Response to: Attitudes ANd FACTORS CONTRIBUTING TO ATTRITION IN CANADIAN SURGICAL SPECIALTY RESIDENCY PROGRAMS}

"Attitudes and factors contributing to attrition in Canadian surgical specialty residency programs" by Adams and colleagues ${ }^{1}$ is of special interest to all final-year medical students, like myself, currently applying for surgical residencies in Canada.

The authors point out that most residents leaving general surgery programs pursue nonsurgical fields. This is consistent with my own anecdotal survey of colleagues in a variety of surgical specialties who left their residencies for family practice. The study suggests an underestimation by medical students who go on to pursue surgical residencies of the disparity between work life and personal life that is inherent to becoming a highly competent surgeon. Interestingly, the article also highlights that surgical residency attrition cannot be purely because of long hours and poor lifestyle. The questions posed, if reframed by medical students considering surgical specialties, queries, "Do we really know what we are getting ourselves into?"

Three main points come to mind. First, an important factor that is widely known and canvassed is the lack of meaningful exposure to most of the surgical residency choices during medical school, which may play a role in later regrets and attrition. The clerkship year introduces medical students to the ward, core areas of medicine, and some limited subspecialized areas. Students rotate through the various specialties as if throuhg a restaurant's tasting menu, with each exposure spanning a mere few weeks. The Canadian Resident Matching Service (CaRMS) deadline in November of final year, just 6 months after clerkship ends, forces students to restrict the number and types of elec- tives that can be accommodated in the small window before applications are due. Experiences encountered after the deadline may have limited influence on choice of career. One only hopes students do not discover their calling during a winter or spring elective during final year, when the ship for applying has already sailed. It cannot be surprising that young physicians find themselves in an area of medicine they are unhappy with or not particularly suited for, and that they ultimately feel unfulfilled.

Second, it is widely understood that medical students are expected to demonstrate "commitment" to their surgical specialty of choice. ${ }^{2,3}$ Thus, students often further prune their limited pre-CaRMS electives to be a competitive applicant. This is a great shame because, although the days of the rotating internship are long gone, many a satisfied and happy senior doctor have divulged that they have found themselves in an unintended area after rotating through a fortuitous elective.

Third, fear of underemployment is real and palpable among students, and why wouldn't it be? This is a relatively recent concern for the medical profession and a phenomenon the previous generation didn't face.

At the end of the day, I have always been told that your best track is the work you love to do - if indeed you are lucky enough to discover that early enough in your training.

\section{Julia B. Lichtenstein, BSc, AAS}

Affiliation: From the University of British Columbia, Vancouver, BC.

\section{DOI: $10.1503 /$ cjs. 1760062}

\section{References}

1. Adams S, Ginther DN, Neuls E, et al. Attitudes and factors contributing to attrition in Canadian surgical specialty residency programs. Can 7 Surg 2017;60: 247-52.
2. Marwan Y, Waly F, Algarni N, et al. The role of letters of recommendation in the selection process of surgical residents in Canada: a national survey of program directors. 7 Surg Educ 2017;74:762-7.

3. Krauss E, Bezuhly M, Williams J. Selecting the best and brightest: a comparison of residency match processes in the United States and Canada. Plast Surg 2015;23: 225-30.

\section{AUTHOR RESPONSE}

We are grateful to Ms. Lichtenstein for taking the time both to read our paper and for offering her thoughts and observations on the issues raised.

The purpose of our study was partly to try to quantify the scale of the problem of career dissatisfaction in surgery at the resident level, but also to create some food for thought among those on either side of the Canadian Resident Matching Service (CaRMS) process. The observation that it is difficult to confidently recognize what one will want to be doing for the next 30 or 40 years based on a few weeks of limited exposure is well recognized in the world of medical education. This appears to be equally true whether one has entered medical school directly from high school, as is the European model, or after a period of undergraduate tertiary education, as is required in North America. Indeed changes in preferences, values and life goals can occur at any age, not least in the early stages of one's career regardless of the field or vocation.

Ultimately the best advice we can offer to those entering the CaRMS match is to do everything you can to "know what you're getting yourselves into." This is, after all, why students are favoured if they have shown a commitment to a specialty - not that it constitutes a guarantee of subsequent fulfilment and happiness, but because it is simply an indication that they have as good an idea of what they're getting themselves into as they can possibly get for their stage in 
life. Further academic research into medical students' career decisionmaking processes and their level of appreciation into what a career in the various specialties entails would undoubtedly be of value to both medical students and postgraduate educators alike. Unfortunately, with medical education being as demanding and intensive as it is and with the volume of knowledge that must be instilled in this timeframe there can only ever be a short, finite window of opportunity in which medical students can consider and research their potential future careers. On that rather poignant note we wish Ms. Lichtenstein and all those entering CaRMS the very best of luck in matching to the specialty to which they are most suited.

\section{Simon T. Adams, MBChB, MD; D. Nathan Ginther, MD; Evan D. Neuls, MD; Paul Hayes, MD}

Affiliations: From the University of Saskatchewan, Saskatoon, Sask.

DOI: $10.1503 /$ cjs. 1760063

Medical students interested in the various surgical topics published in the Canadian Journal of Surgery can subscribe to the Table of Contents email: https://canjsurg.ca/alerts/

\section{Evaluation OF THE INFLUENCE OF STUDENT-LED SURGERY INTEREST GROUPS: A PAN-CANADIAN SURVEY}

Interest in surgical residency programs has been decreasing in Canada and the United States over the past 2 decades. While this phenomenon is likely multifactorial, early surgical exposure and surgical role models are factors known to increase the number of applications to surgical residency programs. Surgery interest groups (SIGs) have arisen as a student-led initiative to foster interest in aspiring medical students and provide a platform to challenge dissuasive longstanding notions surrounding worklife balance and personal satisfaction.

A number of papers recently published in the Canadian Fournal of Surgery $(C 7 S)$ have addressed the topics of surgical education, recruitment, and residency attrition rates in Canada. ${ }^{1-3}$ These studies unanimously discuss the importance of early surgical exposure to improve continuity in both entrance and completion rates of surgical disciplines. Adams and colleagues ${ }^{3}$ resonate this notion: "efforts to educate prospective residents about the reality of the surgical lifestyle and to optimize employment prospects may improve [residency] completion rates."

We conducted a pan-Canadian study investigating how SIGs operate and influence medical students' interest in surgical careers. The study was completed by disseminating 2 unique surveys to SIG executive and members enrolled in Canadian medical schools during the 2016/17 academic year. The executive survey focused on types of events hosted, the structure/ support of their society, as well as barriers and plans for improvement. The members survey focused on degree of student involvement, impact on interest and competencies, and avenues for improvement.

The key findings from our survey showed that surgical skills events followed by career nights were the most anticipated and beneficial to members (Fig. 1). The largest barriers to implementing SIG initiatives were insufficient funding and time conflicts with other student groups. Hence, increased budgets and administrative aid were deemed the greatest opportunities for improvement. Conversely, member survey respondents commonly felt SIG events were collegial and accessible opportunities to complement their academic curricula. Overall, members felt that SIGs provided meaningful preclinical expo- sure, helped develop connections and mentorship, and addressed inquiries surrounding occupation and lifestyle to fortify interest in surgical careers.

Previous studies investigating the influence of surgical societies to pique surgical interests have also illustrated greater rates of interest and enrolment in surgical disciplines. Namely, a Columbia University-based study ${ }^{4}$ found that entrance rates into general surgery programs tripled following establishment of their SIG. The existing literature surrounding the utility and practicality of SIGs highlights their role in encouraging future generations of medical students toward a career in surgery.

Early surgical exposure through SIGs and similar student-led initiatives shows clear promise in achieving the common goal of fostering exploration-driven interest in a historically daunting field. The ongoing support of undergraduate medical education departments is necessary for SIGs to thrive and help meet the persistent demand for surgeons across Canada.

\section{Jin Soo Andy Song, PhD}

Affiliation: From the Faculty of Medicine, Dalhousie University, Halifax, NS.

DOI: 10.1503/cjs.013017

\section{References}

1. Gentles JQ. Why wait until residency? Competency-based education in longitudinal integrated clerkships. Can 7 Surg 2017;60:64-5.

2. Strelzow J, Petretta R, Broekhuyse M. Factors affecting orthopedic residency selection: a cross-sectional survey. Can $\mathcal{F}$ Surg 2017;60:186-91.

3. Adams S, Ginther DN, Neuls E, et al. Attitudes and factors contributing to attrition in Canadian surgical specialty residency programs. Can 7 Surg 2017;60: 247-52.

4. Salna M, Sia T, Curtis G, et al. Sustained Increased entry of medical students into surgical careers: a student-led approach. 7 Surg Educ 2016;73:151-6. 